\title{
ON THE NON-VANISHING OF POINCARÉ SERIES
}

\author{
by C. J. MOZZOCHI
}

(Received 18th August 1987)

R. A. Rankin [2] and J. Lehner [1] considered the non-vanishing of Poincare series for the classical modular matrix group and for an arbitrary fuchsian group, respectively.

In this paper we consider the non-vanishing of Poincaré series for the congruence group

$$
\Gamma=\Gamma_{0}(N)=\left\{\left(\begin{array}{ll}
a & b \\
c & d
\end{array}\right) \equiv\left(\begin{array}{ll}
* & * \\
0 & *
\end{array}\right)(\bmod N)\right\} ; N \geqq 1 .
$$

For $k>2, k \equiv 0(\bmod 2)$, let $\mathscr{M}_{k}^{0}(\Gamma)$ be the space of cusp forms for $\Gamma$ of weight $k$. Let $\mu_{k}$ be the dimension of $\mathscr{M}_{k}^{0}(\Gamma)$. Let

$$
P_{m}(z, k)=\sum_{\gamma \in \Gamma_{\infty} \backslash \Gamma}(j(\gamma, z))^{-k} e(m \gamma z),
$$

where

$$
j(\gamma, z)=c z+d \quad \text { if } \quad \gamma=\left(\begin{array}{ll}
* & * \\
c & d
\end{array}\right)
$$

and

$$
e(z)=e^{2 \pi i z}
$$

be the Poincaré series of weight $k$ attached to $\Gamma$. The space $\mathscr{H}_{k}^{0}(\Gamma)$ is spanned by $P_{m}(z, k)$. Since $\mathscr{M}_{k}^{0}(\Gamma)$ is finite dimensional, there must be many linear relations between $P_{m}(z, k)$. Very little is known about these relations. In particular one does not know which $P_{m}(z, k)$ do not vanish identically.

In the case of full modular group $\Gamma=\Gamma_{0}(1)$, when $k=4,6,8,10$ and $14, \mathscr{M}_{k}^{0}(\Gamma)$ has dimension zero; so that $P_{m}(z, k)$ vanishes identically for all positive integers $m$. We have $\mu_{k}>0$ for $k=12$ and all $k \geqq 16$. Indeed by Theorem 6.1.2 in [3] we have for $k \geqq 4$,

$$
\mu_{k}= \begin{cases}{\left[\frac{k}{12}\right]} & \text { if } k \neq 2(\bmod 12), \\ {\left[\frac{k}{12}\right]-1} & \text { if } k \equiv 2(\bmod 12) .\end{cases}
$$


Clearly, since $P_{m}(z, k)\left(1 \leqq m \leqq \mu_{k}\right)$ span the space $\mathscr{M}_{k}^{0}(\Gamma)$, we have $P_{m}(z, k) \not 0$ for $1 \leqq m \leqq \mu_{\mathbf{k}}$. Rankin [2] was able to show that many more Poincare series do not vanish.

In this paper we extend the arguments of Rankin to establish:

Theorem 1. For $\Gamma=\Gamma_{0}(N) ; N \geqq 1$, we have $P_{m}(z, k) \equiv 0$ if

$$
m(m, N) \alpha^{2}(m) \leqq \frac{1}{2^{15} \pi^{3}}\left(\frac{N}{\tau(N) \log 2 N}\right)^{2}
$$

where

$$
\alpha(m)=\sum_{d \mid m} \frac{\tau(d)}{\sqrt{d}}
$$

and $\tau(N)$ is the number of positive divisors of $N$.

Remarks. Stripped of factors of lower order, Theorem 1 states essentially that

$$
m(m, N) \leqq K(N / \log N)^{2},
$$

where $K$ is an explicitly defined numerical constant and $K<1$. Thus, for small values of $N$, where the right hand side is less than 1, this tells us nothing. Even for large $N$ it is vacuous in some cases, e.g., when $N$ divides $m$, as it then gives $m / N<K(\log N)^{-2}$. However, in other cases it will give information. For example, whenever

$$
N / \log N>K^{-1 / 2}
$$

it tells us that, for all $k>2$, the first Poincare series does not vanish.

Note also that, unlike the results of Rankin and Lehner, the upper bound does not depend on the weight $k$. However, in Theorem 2 and Theorem 3, which follow, the upper bound does depend on the weight $k$.

Let $S(m, m ; c)$ be the Kloosterman sum defined

$$
S(m, m ; c)=\sum_{d(\bmod c)}^{*} e\left(m \frac{d+d}{c}\right) ; d \bar{d} \equiv 1(\bmod c) .
$$

Let $J_{k-1}(y)$ be the Bessel function of order $k-1$.

Lemma 1. (A. Weil cf. [4]). We have

$$
|S(m, m ; c)| \leqq(m, c)^{1 / 2} c^{1 / 2} \tau(c) .
$$

Lemma 2. (cf. [5]). We have 


$$
\left|J_{k-1}(y)\right| \leqq \min \left\{1, \frac{1}{(k-1) !}\left(\frac{y}{2}\right)^{k-1}\right\} \leqq \min \left\{1, \frac{y}{2}\right\}
$$

Proof (of Theorem 1).

By the argument presented in Section 2 of [2], and in Chapter 5 of [3], we have

$$
P_{m}(z, k) \equiv 0 \quad \text { if } \quad\left|S_{m}\right|<\frac{1}{2 \pi}
$$

where

$$
S_{m}=\sum_{r=1}^{\infty}(r N)^{-1} S(m, m ; r N) J_{k-1}\left(\frac{4 \pi m}{r N}\right)
$$

Clearly, by Lemma 1 and Lemma 2, we have

$$
\begin{aligned}
\left|S_{m}\right| & \leqq \frac{(m, N)^{1 / 2} \tau(N)}{N^{1 / 2}} \sum_{r=1}^{\infty} \frac{(m, r)^{1 / 2} \tau(r)}{r^{1 / 2}} \min \left\{1, \frac{2 \pi m}{r N}\right\} \\
& \leqq \frac{(m, N)^{1 / 2} \tau(N)}{N^{1 / 2}} \sum_{d \mid m} \tau(d) \sum_{r=1}^{\infty} \frac{\tau(r)}{r^{1 / 2}} \min \left\{1, \frac{2 \pi m}{r d N}\right\} \\
& \leqq \frac{(m, N)^{1 / 2} \tau(N)}{N^{1 / 2}} \sum_{d \mid m} \tau(d) \sum_{r_{1}=1}^{\infty} \sum_{r_{2}=1}^{\infty} \frac{1}{\left(r_{1} r_{2}\right)^{1 / 2}} \min \left\{1, \frac{2 \pi m}{r_{1} r_{2} d N}\right\} .
\end{aligned}
$$

Let

$$
R=\left(\frac{2 \pi m}{d N}\right)
$$

Then

$$
\begin{aligned}
\sum_{r_{1}=1}^{\infty} \sum_{r_{2}=1}^{\infty} \frac{1}{\left(r_{1} r_{2}\right)^{1 / 2}} \min \left\{1, \frac{R}{r_{1} r_{2}}\right\} & =2 \sum_{r=1}^{\infty} \frac{1}{r^{1 / 2}} \min \left\{1, \frac{R}{r}\right\}+\sum_{r_{1}=2}^{\infty} \sum_{r_{2}=2}^{\infty} \frac{1}{\left(r_{1} r_{2}\right)^{1 / 2}} \min \left\{1, \frac{R}{r_{1} r_{2}}\right\} \\
& =2 S_{1}+S_{2} .
\end{aligned}
$$

Case I: $R>1$.

$$
S_{1} \leqq 1+\int_{1}^{R} t^{-1 / 2} d t+R \int_{R}^{\infty} t^{-3 / 2} d t ; \text { so that }
$$




$$
\begin{gathered}
S_{1} \leqq 4 R^{1 / 2}-1 \leqq 4 R^{1 / 2}(1+\log (R+1)) \\
S_{2} \leqq \int_{1}^{R}\left(\int_{1}^{R / t_{1}}\left(t_{1} t_{2}\right)^{-1 / 2} d t_{2}\right) d t_{1}+R \int_{1}^{R}\left(\int_{R / t_{1}}^{\infty}\left(t_{1} t_{2}\right)^{-3 / 2} d t_{2}\right) d t_{1} \\
+R \int_{R}^{\infty}\left(\int_{1}^{\infty}\left(t_{1} t_{2}\right)^{-3 / 2} d t_{2}\right) d t_{1} ; \text { so that } \\
S_{2} \leqq 4 R^{1 / 2}(1+\log (R+1)) .
\end{gathered}
$$

Case II: $0<R \leqq 1$.

$$
\begin{gathered}
S_{1} \leqq R+R \int_{1}^{\infty} t^{-3 / 2} d t ; \text { so that } \\
S_{1} \leqq R+2 R \leqq 3 R^{1 / 2}(1+\log (R+1)) ; \text { since } R \leqq 1 \\
S_{2} \leqq \int_{1}^{\infty}\left(\int_{1}^{\infty} \frac{R}{\left(t_{1} t_{2}\right)^{3 / 2}} d t_{2}\right) d t_{1} ; \text { so that } \\
S_{2} \leqq 4 R \leqq 4 R^{1 / 2}(1+\log (R+1)) ; \text { since } R \leqq 1
\end{gathered}
$$

By combining both cases with the earlier calculations, the proof is completed.

Theorem 2. Let $\Gamma=\Gamma_{0}(N) ; N \geqq 1$. There exist positive constants $k_{0}$ and $B$ (both independent of $N$ ), where $B>4 \log 2$ such that, for all $k \geqq k_{0}$ and all positive integers $m$ such that

$$
k \leqq m \leqq k^{2} \exp (-B \log k / \log \log k)
$$

$P_{m}(z, k) \neq 0$.

Proof. Let $Q^{*}=(4 \pi m / v N)$.

$$
\left|S_{m}\right| \leqq \sum_{1 \leqq q<Q^{*}} \frac{|S(m, m ; q N)|}{q N}\left|J_{k-1}\left(\frac{4 \pi m}{q N}\right)\right|+\sum_{q \geqq Q^{*}} \frac{\mid S(m, m ; q N \mid}{q N}\left|J_{k-1}\left(\frac{4 \pi m}{q N}\right)\right| .
$$

$\left|S_{m}\right| \leqq S_{m}^{\prime}+S_{m}^{\prime \prime}$

Clearly, 


$$
S_{m}^{\prime} \leqq \sum_{1 \leqq q<Q^{*} N=Q} \frac{|S(m, m ; q)|}{q}\left|J_{k-1}\left(\frac{4 \pi m}{q}\right)\right|,
$$

where $Q$ is defined in [2]. Hence by exactly the same argument presented in [2], we have

$$
S_{m}^{\prime} \leqq A_{6} M(m)\left\{\sigma^{6} m^{1 / 2} \sigma_{-1 / 2}(m)+(4 \pi)^{1 / 2} \sigma^{2} \sigma_{0}(m)\right\} .
$$

Clearly, by the argument presented in [2],

$$
S_{m}^{\prime \prime} \leqq \sum_{q \geqq Q^{*}}\left|J_{v}\left(\frac{v Q^{*}}{q}\right)\right| \leqq A_{5} \sum_{q \geqq Q^{*}} f\left(\frac{Q^{*}}{q}\right) \leqq A_{5}\left\{Q^{*} \int_{0}^{1} x^{-2} F(x) d x+\sigma^{2}\right\}
$$

so that, since $Q^{*} N=Q$,

$$
S_{m}^{\prime \prime} \leqq A_{5} \sigma^{2}+\frac{A_{7} m \sigma^{15}}{N}\left(\frac{1}{2} e x_{0}\right)^{v}+\frac{A_{8} m \sigma^{12}}{N} \leqq A_{5} \sigma^{2}+A_{9} m \sigma^{12}
$$

Hence $\left|S_{m}\right| \leqq A_{6} m^{1 / 2} \sigma^{6} M(m) \sigma_{-1 / 2}(m)+A_{10} \sigma^{2} M^{2}(m)+A_{5} \sigma^{2}+A_{9} m \sigma^{12}$, and the result follows by the argument presented in [2] with the obseration that $A_{5} \sigma^{2}=o(1)$.

Theorem 3. For $\Gamma=\Gamma_{0}(N) ; N \geqq 1$, we have $P_{m}(z, k) \not 00$ if $k_{0}(N) \leqq k$ and for any $\varepsilon>0$

$$
m^{1+\varepsilon}(m, N) \alpha^{2}(m) \ll\left(\frac{N k}{\tau(N)}\right)^{2} .
$$

Proof. By Lemma 1 we have

$$
S_{m}^{\prime} \leqq \frac{(m, N)^{1 / 2} \tau(N)}{N^{1 / 2}} \sum_{1 \leqq q<Q^{*}} \frac{(m, q)^{1 / 2} \tau(q)}{q^{1 / 2}}\left|J_{k-1}\left(\frac{4 \pi m}{q N}\right)\right| .
$$

Clearly,

$$
\begin{gathered}
S_{m}^{\prime} \leqq \frac{(m, N)^{1 / 2} \tau(N)}{N^{1 / 2}} \sum_{\substack{d \mid m \\
d \leqq Q^{*}}} \tau(d) \sum_{1 \leqq r<\left(Q^{* / d}\right)} \frac{\tau(r)}{r^{1 / 2}}\left|J_{k-1}\left(\frac{4 \pi m}{r d N}\right)\right| \\
S_{m}^{\prime} \ll \frac{(m, N)^{1 / 2} \tau(N) m^{\varepsilon}}{N^{1 / 2} Q^{* 1 / 2}} \sum_{\substack{d / m \\
d^{*} Q^{*}}} \tau(d) d^{1 / 2} S_{d}, \text { where } \\
S_{d}=\sum_{1 \leqq r<\left(Q^{*} / d\right)}\left(\frac{Q^{*}}{r d}\right)^{1 / 2}\left|J_{v}\left(\frac{v Q^{*}}{r d}\right)\right| .
\end{gathered}
$$


By the same argument presented in [2], we have

$$
S_{d} \ll\left(\frac{m \sigma^{9}}{N d}+\sigma^{2}\right) \text {. }
$$

Hence

$$
S_{m}^{\prime \ll} \frac{(m, N)^{1 / 2} \tau(N) m^{1 / 2+\varepsilon}}{N(k-1)}\left(\sum_{\substack{d \mid m \\ d<Q^{*}}} \frac{\tau(d)}{d^{1 / 2}}\right)+(m, N)^{1 / 2} \tau(N) m^{\varepsilon}(k-1)^{1 / 6} \sum_{\substack{d \mid m \\ d<Q^{*}}} \tau(d)\left(\frac{d}{m}\right)^{1 / 2} .
$$

But

$$
\sum_{\substack{d \mid m \\ d<Q^{*}}} \frac{\tau(d)}{d^{1 / 2}} \leqq \alpha(m)
$$

and since $d<Q^{*}$ we have

$$
\sum_{\substack{d \mid m \\ d<Q^{*}}} \tau(d)\left(\frac{d}{m}\right)^{1 / 2} \ll \sum_{d \mid m} \tau(d)\left(\frac{1}{(v N)^{1 / 2}}\right) \ll \frac{m^{2 \varepsilon}}{(v N)^{1 / 2}} .
$$

Hence

$$
S_{m}^{\prime} \ll \frac{(m, N)^{1 / 2} \tau(N) m^{1 / 2+\varepsilon} \alpha(m)}{N(k-1)}+\frac{(m, N)^{1 / 2} \tau(N) m^{3 \varepsilon}(k-1)^{1 / 6}}{N^{1 / 2}(k-1)^{1 / 2}} .
$$

By the argument given in the proof of Theorem 2, we have $S_{m}^{\prime \prime} \leqq A_{5} \sigma^{2}+A_{9} m \sigma^{12}$. Hence

$$
S_{m} \ll \frac{(m, N)^{1 / 2} \tau(N) m^{1 / 2+\varepsilon} \alpha(m)}{N(k-1)}+\frac{(m, N)^{1 / 2} \tau(N) m^{3 \varepsilon}}{N^{1 / 2}(k-1)^{1 / 3}}+\frac{1}{(k-1)^{1 / 3}}+\frac{m}{(k-1)^{2}},
$$

and the result follows from the hypothesis; since the last three terms are sufficiently small for $k>k_{0}(N)$.

Note added in proof. All of the results in this paper are also true for the principal congruence groups $\Gamma(N) ; N \geqq 1$.

\section{Acknowledgements.}

I would like to thank Professor Henryk Iwaniec for several helpful conversations concerning this problem, and I would like to express my appreciation to the referee for helpful suggestions concerning exposition.

This work was done during the summer of 1987 , and I would like to thank the Institute for Advanced Study for providing me with excellent working conditions. 


\section{REFERENCES}

1. J. Lehner, On the non-vanishing of Poincaré series, Proc. Edinburgh Math. Soc. 23 (1980), 225-228.

2. R. A. Rankin, The vanishing of Poincaré series, Proc. Edinburgh Math. Soc. 23 (1980), 151-161.

3. R. A. Rankin, Modular Forms and Functions (Cambridge, 1977).

4. A. WeIl, On some exponential sums, Proc. Acad. Sci. U.S.A. 34 (1948), 204-207.

5. G. N. Watson, A Treatise on the Theory of Bessel Functions (Cambridge, 1922).

School of Mathematics

InSTITUTE for Advanced StUdy

Princeton, NJ 08540 